\title{
Genesis
}

Manuscrits - Recherche - Invention

$53 \mid 2021$

Sartre Beauvoir. Genèses croisées

\section{Marcel Proust, Les Soixante-quinze Feuillets et autres \\ manuscrits inédits, édition établie par N. Mauriac Dyer, préface de Jean-Yves Tadié, Paris, Gallimard, coll. «Blanche », 2021, 380 p.}

\section{Ilaria Vidotto}

\section{OpenEdition}

\section{Journals}

Édition électronique

URL : https://journals.openedition.org/genesis/6382

DOI : $10.4000 /$ genesis.6382

ISSN : 2268-1590

Éditeur :

Presses universitaires de Paris Sorbonne (PUPS), Société internationale de génétique artistique littéraire et scientifique (SIGALES)

\section{Édition imprimée}

Date de publication : 15 décembre 2021

Pagination : 214-216

ISBN : 979-10-231-0716-6

ISSN : $1167-5101$

\section{Référence électronique}

Ilaria Vidotto, «Marcel Proust, Les Soixante-quinze Feuillets et autres manuscrits inédits, édition établie par N. Mauriac Dyer, préface de Jean-Yves Tadié, Paris, Gallimard, coll. « Blanche », 2021, 380 p. », Genesis [En ligne], 53 | 2021, mis en ligne le 15 décembre 2021, consulté le 13 janvier 2023. URL : http://journals.openedition.org/genesis/6382 ; DOI : https://doi.org/10.4000/genesis.6382 
Jacinthe Martel, professeur au département d'études littéraires de l'université du Québec à Montréal, où elle mène une recherche consacrée aux archives des écrivains.

Commençons par signaler que cette bibliothèque n'est que sa dernière bibliothèque, celle qu'il a disposée dans sa maison de la Petite-Bourgogne à partir de 1992. En outre, elle ne rend compte que d'une partie de ses lectures puisque Ducharme empruntait beaucoup. Elle est par ailleurs hétérogène puisque, comme Michel Aquin partageant ses livres avec Andrée Yanacopoulo, Ducharme y conservait des livres appartenant à Claire Richard, comédienne et auteure de séries dramatiques pour la télévision, qui fut sa compagne, son agente et son porte-parole dès leur rencontre en 1967. Inversement, certains ouvrages appartenant à Réjean Ducharme étaient rangés dans la bibliothèque de Claire Richard. Il faut enfin faire la part du hasard. Une partie des livres et des disques de la bibliothèque est issue de glanages impromptus constitués lors de balades à pied ou à vélo. Ducharme y accumule également des objets, qui font parfois office d'appuie-livres : appareil-photo, canard en plastique, tête de poupée, aimants de haut-parleurs... De nombreux volumes contiennent entre deux pages des collages, des fleurs séchées ou des feuilles d'arbre. Les photographies de Monique Bertrand qui ponctuent le présent ouvrage rendent compte de ces curiosités avec un sens aigu de l'objet.

Qu'en est-il donc de cet inventaire? Bouclé dans l'été 2018, il suit la disposition des livres dans la maison de l'écrivain. Chaque ouvrage s'est vu attribuer une cote alphanumérique, permettant de l'identifier par le nom de l'auteur et par la place occupée dans la géographie intime. La première section recense les livres conservés dans le bureau; ils sont disposés sur six grandes étagères en acier, sur la table de travail couverte de dictionnaires, de lexiques et d'encyclopédies -, ainsi que sur le bord des fenêtres. Les livres dispersés dans la maison ont été regroupés dans la section II. La section III comprend les «livres de chevet». En annexes, figurent les livres téléchargés et les ouvrages issus d'emprunts à des bibliothèques publiques. La cote $\mathrm{A} 1.1$, qui donne son titre au présent ouvrage, correspond dans cette nomenclature au premier livre rangé sur l'étagère $\mathrm{A}$ du bureau (en l'occurrence La Vie littéraire, d'Anatole France). Systématiquement mentionnées également, les annotations figurant dans les ouvrages (phrases ou fragments rédigés), les notes de lecture (mots ou listes de mots suivis de numéros de page) et les rares marginalia (soulignements, traits dans les marges).

Cet inventaire sera utilement complété pour les chercheurs par un inventaire photographique inspiré du projet «Bacon's Books : Francis Bacon's Library and its Role in his Art», réalisé par Monique Bertrand, bientôt disponible en ligne, qui comprendra «les couvertures des livres, les pages de titre et celles qui portent diverses marques (dédicace, signature, signet, etc.), les reliures, les traces d'usure, de colle ou de peinture, le feuilleté des dictionnaires laissés ouverts sur la table du bureau, les éditions particulières etc. » Et last but not least, l'analyse de cette bibliothèque devra tenir le plus grand compte des notes de lecture rédigées par Réjean Ducharme, un ensemble très riche, inédit, qui sera bientôt déposé dans le fonds Réjean Ducharme de la BAC, LMS-0130.

Marcel Proust, Les Soixante-quinze Feuillets et autres manuscrits inédits, édition établie par N. Mauriac Dyer, préface de Jean-Yves Tadié, Paris, Gallimard, «Blanche», 2021, 380 p.

\section{Compte rendu par Ilaria Vidotto*}

«Le moment sacré»: c'est avec cette formule hyperbolique que Jean-Yves Tadié a choisi d'intituler sa préface à l'une des publications les plus retentissantes de l'année 2021 : Les Soixante-quinze feuillets et autres manuscrits inédits de Marcel Proust, parus chez Gallimard dans une édition établie, remarquablement, par Nathalie Mauriac Dyer. Le titre de Tadié aurait pu d'ailleurs figurer au pluriel, puisqu'il y eut au moins deux moments sacrés : celui, tout récent, de la prise de connaissance de ces inédits par la communauté des proustiens et des lecteurs, après plus d'un demi-siècle de spéculations et de mystères; et celui, plus éloigné, de la fin de l'année 1907, date à laquelle remonte, en toute vraisemblance, le début de la rédaction de ces fragments. Sans le savoir, Marcel Proust découvre, dans ces ébauches qui le ramènent au roman après l'échec de Jean Santeuil et la parenthèse des traductions ruskiniennes, le noyau matriciel de ce «long ouvrage ${ }^{5}$ » à venir qui s'appellera À la recherche du temps perdu. Les lecteurs d'aujourd'hui découvrent, quant à eux, un chaînon encore manquant dans l'histoire complexe et passionnante de la genèse du roman, les fondations soupçonnées, et cachées jusque-là, de la cathédrale proustienne.

Soupçonnées, ces fondations l'étaient au moins depuis 1954; Bernard de Fallois, qui avait eu accès aux nombreux manuscrits conservés dans les archives de la nièce de l'écrivain, Suzy Mante-Proust, avait en effet mentionné l'existence de ce groupe de textes dans la préface à son édition des inédits réunis pour la première fois sous le titre Contre Sainte-Beuve 6 . Or, contrairement à cet ensemble, contrairement aussi à l'importante masse de brouillons constituant le manuscrit de Jean Santeuil, ces fameux 75 feuillets (76, pour être exact 7 ), ainsi que quelques nouvelles plus anciennes ${ }^{8}$ et

(*) Université de Lausanne.

5. Marcel Proust, Correspondance, t. IX, éd. Ph. Kolb, Paris, Plon, 1982, p. 221

6. Voir Marcel Proust, Contre Sainte-Beuve (1954), éd. B. de Fallois, Paris, Gallimard, coll. «Folio essais », 1987, p. 11-12.

7. Les feuillets sont en effet 76, mais l'éditrice a choisi de maintenir l'appellation sous laquelle le manuscrit a été mentionné par Bernard de Fallois en 1954, voir «Notes sur la présente édition», p. 18, note 1 .

8. Il s'agit d'un groupe de nouvelles prévues initialement pour faire partie du recueil des Plaisirs et les Jours mais finalement retranchées par Proust ; elles ont été publiées en 2019 par les éditions De Fallois, dans une édition de Luc Fraisse, voir Le Mystérieux Correspondant et autres nouvelles inédites, Paris, De Fallois, 2019. 
d'autres morceaux appartenant au même «entourage» génétique des 75 feuillets ${ }^{9}$, n'ont jamais été livrés au public, De Fallois les ayant gardés religieusement - d'autres diraient jalousement - par-devers lui jusqu'en 2018, année de son décès. Les raisons véritables de cette «occultation» resteront à jamais inconnues, et la présente édition, prenant sur ce point épineux de l'histoire le parti de la discrétion, n'apporte hélas pas d'informations décisives.

Quoi qu'il en soit, les 75 feuillets ont perdu désormais leur aura fantomatique et acquis une matérialité que donnent pour l'instant à imaginer seulement les notices accompagnant la transcription du texte - en attendant la mise à disposition du fac-similé sur le site de Gallica ${ }^{10}$. Le généticien lira ainsi avec intérêt la description soignée du manuscrit fournie par Nathalie Mauriac Dyer (p. 16-17) : on y apprend, entre autres, que les 75 feuillets se présentent sous la forme de 43 bifeuillets de papier vélin non réglé de format $360 \times 230 \mathrm{~mm}$, et qu'ils constituent un ensemble non négligeable de 172 pages écrites. Outre les précisions concernant l'état de conservation du manuscrit, l'absence de pagination, la présence de feuilles vierges et de quelques croquis de la main de Proust, l'éditrice explique comment certains feuillets ont dû être reclassés ou différemment répartis par rapport aux groupements originaires effectués par De Fallois ; de même, les intitulés attribués initialement à chaque groupe de textes n'ont été qu'en partie maintenus, Mauriac ayant par exemple remplacé, conformément aux connaissances établies sur la genèse du roman, le titre «soirées de Combray », donné par De Fallois au premier ensemble, par le plus satisfaisant «une soirée à la campagne» 11 .

De tels détails visent évidemment à compenser l'absence d'une reproduction du manuscrit original, et à satisfaire, du moins partiellement, la curiosité et les exigences des généticiens. Ces derniers se trouvent en effet confrontés à une transcription fortement allégée, «toilettée» de presque toutes les traces matérielles du processus d'écriture. Pour ce qui est, précisément, des choix de présentation et d'établissement du texte, on peut considérer que l'éditrice a réussi un pari qui était loin d'être gagné : satisfaire, d'une part, aux attendus de rigueur philologique que partagent les spécialistes, conscients des implications scientifiques ainsi qu'éditoriales sous-tendant le travail de reconstitution génétique; répondre, d'autre part, aux besoins de lisibilité et d'accessibilité d'une édition qui, accueillie dans la collection «Blanche» de Gallimard, s'adresse aussi à un lectorat plus vaste. La conciliation de ces deux contraintes a donc abouti à une transcription courante ${ }^{12}$, épurée des ratures (les passages ou mots raturés jugés significatifs sont néanmoins donnés en note), intégrant sans signalement les ajouts de la main de Proust et corrigeant également, au besoin, l'orthographe, les accords fautifs ou la ponctuation irrégulière de l'auteur. La simplification du protocole de transcription ne masque pas pour autant le caractère inachevé du manuscrit - les interruptions sont signalées par un bref commentaire entre crochets - et ne renonce pas non plus à fournir des informations génétiques minimales, peu gênantes à la lecture, comme le passage au folio suivant (pointé par deux barres obliques), la numérotation du feuillet indiquée dans la marge, ou le marquage des surcharges par une barre oblique.

L'appareil de notes a été conçu dans la même logique : apporter des compléments d'information riches et éclairants, sans alourdir la lecture cursive du texte. C'est pourquoi l'éditrice a séparé les notes de bas de page, explicatives et/ou génétiques - elles signalent la leçon du manuscrit si celle-ci n'a pas été conservée dans la transcription, ou bien les leçons légèrement différentes proposées par De Fallois - des notes critiques. Ces dernières se trouvent rejetées en fin de volume, sans appel dans le corps du texte; pour chaque section de l'ensemble, elles fournissent des renseignements d'ordre essentiellement génétique, parfois lexicographique ou biographique, et retracent le trajet évolutif de la scène, du motif ou du personnage commentés. Très détaillées, ces précisions livrent à tout lecteur un excellent aperçu de la complexité du travail de création proustien, alimenté par les recyclages, circulations, déplacements et transformations d'un matériau qui ne cesse de se modifier au fil des rédactions. On précisera enfin que le volume est enrichi en appendice d'une bibliographie critique, d'un index des noms de personnes, de lieux et de personnages, et surtout d'un très utile tableau des concordances entre les passages de la Recherche (donnés dans l'édition de la «Bibliothèque de la Pléiade») et les textes des 75 feuillets qui en forment le soubassement.

Au-delà des aspects proprement matériels et éditoriaux de l'entreprise, qu'est-ce que ces inédits viennent apporter de plus, ou de nouveau, à l'étude de l'œuvre de Proust? Le moment sacré de la découverte est-il à la hauteur d'attentes si longuement entretenues, ou éprouve-t-on plutôt un sentiment, très proustien d'ailleurs, de déception, dès lors que la légende laisse la place à la réalité ? Tout est affaire de points de vue, dirait encore le romancier de la Recherche. En effet, la condition paradoxale que pointe Tadié dans sa préface - le paradoxe consistant en ceci que «ce qui est lu en dernier a été écrit en premier» (p. 13) -, peut susciter chez le lecteur d'inédits deux réactions contraires, sans doute coprésentes.

On peut, d'une part, s'étonner devant tout ce qui, dans les 75 feuillets, diffère du roman «achevé»; être sensible à «la rumeur des distances traversées ${ }^{13}$ » en constatant

9. C'est-à-dire les «Autres manuscrits inédits » qui suivent, dans cette édition, les 75 feuillets, voir p. 115-135.

10. Â l'instar des autres manuscrits qui constituent le fonds Proust de la BnF, recensés sur le site de l'équipe Proust de l'ITEM : http://www. item.ens.fr/fonds-proust-numerique/.

11. Le titre donné par De Fallois est anachronique, dans la mesure où on sait que le toponyme «Combray » n'apparaît dans les cahiers de brouillons qu'en 1909, dans le cahier 4.

12. Précisons que la transcription diplomatique, établie par Nathalie Mauriac Dyer, avec la collaboration de Bertrand Marchal, sera disponible sur le site de Gallimard.

13. Marcel Proust, Du côté de chez Swann, À la recherche du temps perdu, t. I, Paris, Gallimard, coll. «Bibliothèque de la Pléiade», 1987, p. 46. 
combien de tâtonnements, de refontes et de transmutations ont été nécessaires pour parvenir au texte que l'on connaît. À ce propos, l'embryon de «Combray» que constitue le premier fragment, «Une soirée à la campagne», permet de saisir la matrice encore fortement autobiographique du récit proustien. Le microcosme familial dépeint dans ces pages reste très proche de la réalité - en témoignent les prénoms de la mère, Jeanne, de la grand-mère, Adèle, du frère, Robert, qui figurent pour la première et unique fois ici en toutes lettres -, et les personnes, surtout les plus aimées, n'ont pas encore subi ce processus de mythisation qui les transformera en personnages de fiction. La grand-mère peut ainsi, dans ces feuillets, écrire des lettres cryptées et ridicules (p. 28-30), ou faire preuve de taquinerie; la mère être à la fois cruelle et pathétique (saisissante est, à ce propos, l'évocation onirique de son visage profané de mourante, p. 41); le frère Robert apparaître dans la surprenante scène d'adieu au petit chevreau, et exister ainsi au moins une fois avant de disparaître à jamais du roman; le narrateur lui-même ressentir, enfin, des pulsions agressives, presque sadiques, à l'égard de sa mère adorée.

Contrairement à l'homosexualité, qui n'est abordée dans aucun des fragments ${ }^{14}$, la judéité du côté maternel de la famille est thématisée de façon plus évidente, quoique toujours en filigrane (nous renvoyons sur ce point à l'éclairage précieux fourni par l'éditrice dans sa notice, p. 220-227); le personnage du grand-oncle parait également se distancier très peu de l'oncle Weil, même si l'on entrevoit déjà le processus romanesque de diffraction par lequel certains traits de son caractère seront plus tard attribués respectivement à la grand-tante, à Charles Swann et à l'oncle Adolphe. Toujours parmi les différences, on sera surpris de l'absence de développements consacrés à la mémoire involontaire - surprise que rachète pourtant l'émotion de découvrir en une allée d'arbres du village campagnard l'origine de la réminiscence manquée à la base de l'épisode des arbres d'Hudimesnil15. Les souvenirs d'enfance ne sortent donc pas encore d'un morceau de madeleine, bien que les tout premiers avant-textes consacrés à ce sujet capital - qu'on peut lire ici dans le fragment IV des «Autres manuscrits», p. 128-129 - soient contemporains des 75 feuillets. Des traces peuvent néanmoins être décelées en retraçant, comme le fait admirablement Nathalie Mauriac Dyer (p. 232-240), le motif des fleurs d'oranger et de géranium, qui apparaît ici, puis dans les premières rédactions de la réminiscence induite par la tasse de thé, et demeure discrètement associé aux souvenirs des étés à la campagne tout au long des réécritures successives.

En même temps que du «pas encore» révélé par ces inédits - et qui caractérise également la phrase proustienne : pas encore solidement charpentée pour accueillir la complexité des impressions qu'elle est appelée à contenir -, on peut aussi s'émouvoir du «déjà là», des ressemblances et persistances. La scène du baiser maternel, refusé puis obstinément conquis par le héros, se détache en tant qu'événement psychique fondateur; les deux côtés, appelés ici «Villebon» et «Bonneval», sont esquissés avec leurs spécificités géographiques et topographiques, et l'on peut déjà entrevoir le rôle structurant dont ils seront chargés dès les premiers cahiers de 1909. De même, l'univers mondain et amoureux qui gravite autour de la petite station balnéaire qui deviendra, plus tard, le Balbec de la Recherche, prend corps ici à travers l'évocation de la vieille dame timide et snob (snob car maladivement timide), avatar de Mme de Villeparisis, et surtout de la petite bande de jeunes filles hautaines et sportives, qui captivent le héros en raison de leur absolue altérité. Plus confus s'avère en revanche le fragment consacré aux noms nobles, creusets synesthésiques et aimants de rêveries qui viendront ensuite s'agréger dans la lumière orangée du nom de Guermantes ou de Parme. Outre ces permanences thématiques, formant-comme Venise, ici représentée dans sa splendeur troublante de ville où «on habite dans la mer» (p. 105) et où «la personnalité de l'église constituée, délimitée, saisissable en hauteur est étendue en largeur» (p. 108) - les gisements les plus profonds de l'œuvre à venir, les lecteurs avertis reconnaîtront aussi un fonds permanent d'objets, de formules et d'images (le baiser maternel comparé à une hostie, p. 32-33, les carafes plongées dans la rivière, les aubépines), qui surgissent à ce moment et persisteront, inchangées, à travers toutes les couches rédactionnelles. Rien ne se perd, rien ne disparaît vraiment dans l'atelier de Proust, car chaque embryon de style, chaque parcelle de fiction finit par trouver sa place, après bien des détours, dans l'immense édifice du roman.

Ainsi, si l'on ne peut pas affirmer que les 75 feuillets livrent des secrets inouïs au sujet de la Recherche, susceptibles de réviser du tout au tout les connaissances rassemblées au cours d'un siècle d'exégèse, leur lecture est loin d'être décevante. Ce manuscrit est précieux moins par les révélations qu'il contient que par la possibilité qu'il nous offre de contempler les tours et détours d'une imagination créatrice cherchant sa voie, qui essaie et trébuche, mais qui est déjà grosse de trouvailles. N'est-ce pas là, d'ailleurs, l'objet premier de la génétique ? Grâce à la reconstruction impeccable, à la fois érudite et très agréable à lire, fournie par l'appareil critique et éditorial, nous assistons à l'ouverture de l'un des plus vastes chantiers d'écriture de la littérature française. En refermant le volume, avons-nous alors «l'impression de mieux comprendre l'œuvre, qu' on nous explique tout ce qui était caché» (p. 14), comme on lit dans la préface? Non, sans doute, car le roman de Proust gardera toujours ses zones d'ombre, des coins obscurs qui défient l'interprétation; ce que nous comprenons un peu mieux, assurément, c'est le long cheminement de ce chef-d'œuvre inépuisable qu'est la Recherche.

14. L'éditrice identifie néanmoins des évocations allusives tissées dans la description de la bande de fillettes normandes ou dans la trame des toponymes normands qui figurent dans le fragment «Noms nobles», souvenir des excursions de l'été 1907 en compagnie d'Agostinelli. Voir «Notice», p. 240-245.

15. Cet épisode très ancien, présent dans différents avant-textes, se fixera dans À l'ombre des jeunes filles en fleurs. 